\title{
Cytokine production by blood mononuclear cells from in-patients with anorexia nervosa
}

\author{
Esther Nova ${ }^{1}$, Sonia Gómez-Martínez ${ }^{1}$, Gonzalo Morandé ${ }^{2}$ and Ascensión Marcos ${ }^{1}$ * \\ ${ }^{1}$ Instituto de Nutrición y Bromatología (CSIC-UCM), Facultad de Farmacia, Universidad Complutense, Ciudad \\ Universitaria, 28040 Madrid, Spain \\ ${ }^{2}$ Hospital Niño Jesús. C/Menéndez Pelayo 65, 28009 Madrid, Spain
}

(Received 13 September 2001 - Revised 28 February 2002 - Accepted 15 March 2002)

\begin{abstract}
Although protein-energy malnutrition is a common cause of immunodeficiency, the immune function in underweight anorexia nervosa (AN) patients usually seems to be better preserved than would be expected. However, a deranged cytokine production and its consequences are currently being investigated in these patients. This study was aimed at measuring, over time, the capacity of peripheral blood mononuclear cells (PBMC) from AN in-patients to produce several cytokines involved in the regulation of immune responses. The in vitro production of interferon (IFN)- $\gamma$, interleukin (IL)-2, tumour necrosis factor (TNF)- $\alpha$, IL- 6 and IL-1 $\beta$ by phytohaemagglutinin-stimulated PBMC were assessed on forty female adolescents with AN. These measures were carried out twice, upon hospital admission and at discharge, which occurred on average after 1 month. Thirty-five control subjects were also studied. Cytokines were measured by ELISA kits. The production of TNF- $\alpha$ and IL-6 was lower and production of IL-1 $\beta$ higher in AN patients than in the control group at both time points of assessment. Refeeding for 1 month was not enough time to reverse these differences and patients still had a low body weight at discharge. IFN- $\gamma$ production was lower in the patients than in control subjects only at discharge and no differences were found in IL-2 production between both groups. The results suggest that a mechanism involving modifications in the secretion pattern of proinflammatory cytokines could explain some immune function findings in underweight AN patients.
\end{abstract}

\section{Cytokine production: Malnutrition: Anorexia nervosa: Phytohaemagglutinin-stimulated} peripheral blood mononuclear cells

\begin{abstract}
Anorexia nervosa (AN) is characterized by an intense fear of fatness and the individual's refusal to eat with the purpose of losing weight or maintaining it below normal standards. This behaviour is so completely ingrained in the patients that severe malnutrition develops over time and high mortality rates are associated with the illness (Sullivan, 1995).

Nutritional deprivation, such as protein-energy malnutrition, often causes immunodeficiency, leading to increased frequency and severity of infection, thymus atrophy and wasting of peripheral lymphoid tissue (Chandra \& Kumari, 1994). Patients with AN frequently show a tendency to leucopaenia together with relative lymphocytosis (Marcos et al. 1993) and a decreased delayed-type hypersensitivity skin test response (Cason et al. 1986; Varela
\end{abstract}

et al. 1988). However, controversy exists, since in several studies it has been noted that underweight AN patients are surprisingly free from common viral infections (ArmstrongEsther et al. 1978; Golla et al. 1981; Wade et al. 1985). In this regard, consideration should be paid to the nutritional differences between AN and protein-energy malnutrition. The high relative protein intake which is usually found in AN patients, contrary to more typical situations of nutritional deprivation, could contribute to the maintainance of the immune function in underweight $\mathrm{AN}$ patients to a greater extent than would be expected (Marcos, 1997).

Cytokines are among the more important factors regulating immune function and it seems important to address the extent to which these are involved in the pathogenesis of the AN syndrome. Thus, hypotheses have been developed

\footnotetext{
Abbreviations: AN, anorexia nervosa; IFN, interferon; IL, interleukin; PBMC, peripheral blood mononuclear cells; PHA, phytohaemagglutinin; TNF, tumour necrosis factor.

* Corresponding author: Dr Ascensión Marcos, fax +34 91549 5079, email amarcos@eucmax.sim.ucm.es
} 
suggesting cytokines as the fundamental regulators of body metabolism in AN and bulimia nervosa (Holden \& Pakula, 1996). However, it is evident that not all AN patients display the same changes in immune function and cytokine production. A variable lymphocyte proliferative response to different mitogens has been reported in anorectic subjects (Polack et al. 1993). Different results for cytokine production by peripheral blood mononuclear cells (PBMC) have also been reported. For instance, Allende et al. (1998) noted higher spontaneous production of interleukin (IL)-1 by PBMC in AN patients in comparison with a control group, while no differences were observed under similar conditions in other studies (Bessler et al. 1993; Vaisman et al. 1996). Since complex interactions occur between cytokines and the central nervous system, differences in the capacity of AN patients to evoke a compensatory mechanism through either the neuroendocrine system or the autonomic nervous system could explain the variability of the results found.

In order to test the hypothesis that immune function in anorectic individuals is well preserved despite their severely malnourished condition, the present study measured, over time, the capacity of PBMC from AN inpatients to produce several cytokines (interferon (IFN)- $\gamma$, IL-2, tumour necrosis factor (TNF)- $\alpha$, IL-6, IL-1 $\beta$ ) involved in the regulation of the immune response.

\section{Methods and materials}

\section{Subjects}

The subjects in this study were forty female adolescents with AN (age range 13-18 years) recruited upon admission to the Hospital del Niño Jesús for in-patient multidisciplinary treatment. All study individuals met the DSM IV (American Psychiatric Association, 1994) criteria for AN. Twenty-eight of the adolescents were diagnosed with restricting-type AN and twelve with binge-eating-purging type AN. The control group consisted of thirty-five healthy, age-matched female secondary school students with a similar sociocultural background. Control subjects had no history of physical or psychiatric disorders and had not taken any medication for at least 2 months before entering the study. Informed consent to participate in the study was obtained from all seventy-five individuals.

\section{Methods}

Cytokine production by PBMC was assessed twice in the AN group: (1) upon admission to the hospital (AN0); (2) at discharge (AN1), the moment of which was determined by weight recovery criteria together with the evaluation of the psychological condition of the patient. Treatment was provided in an eating-disorders unit, through combined cognitive-behavioural treatment and psychopharmacological medication (ansiolytic drugs (benzodiazepine derivatives) $77.5 \%$ of patients, antidepressant medication (Fluoxetine) $57.5 \%$, antipsychotic drugs $42.5 \%$, several drugs for different gastrointestinal symptoms $15 \%)$. The nutritional support provided a $6.27 \mathrm{MJ}(1500 \mathrm{kcal})$ initial diet and subsequent increases were prescribed until a range of $10 \cdot 46-12.55 \mathrm{MJ} \quad(2500-3000 \mathrm{kcal}) / \mathrm{d}$ was achieved. Three patients were excluded from the analysis, as their data were not available at discharge. At both stages of the study (admission and discharge) basic anthropometric data were obtained by the standard procedure after an overnight fast. Blood was collected in the early morning by puncture of the cubital vein under fasting conditions. Mononuclear cells were isolated from heparinized peripheral blood of patients and controls on Ficoll-Hypaque (Lymphoprep; Nyegaard, Oslo, Norway). Separated PBMC were washed twice with RPMI-1640 medium (BioWhittaker, Verviers, Belgium) and suspended in the same medium, supplemented with fetal bovine serum $(100 \mathrm{ml} / \mathrm{l}$; BioWhittaker) after decomplementation, and containing $1 \%$ penicillin-streptomycin $(5000 \mathrm{IU} / \mathrm{ml}, 5000 \mu \mathrm{g} / \mathrm{ml}$; BioWhittaker). A sample of culture medium $(1 \mathrm{ml})$ containing $10^{6} \mathrm{PBMC}$ was placed into each of four wells of a twenty-four well plate (Becton Dickinson, Sunnyvale, CA, USA). Phytohaemagglutinin (PHA; Gibco BRL, Paisley, UK), at a final concentration of $7 \mathrm{pg} / \mathrm{ml}$, was used as a stimulus in all cases. Cultures were incubated for $48 \mathrm{~h}$ at $37^{\circ} \mathrm{C}$ in a humidified atmosphere containing $5 \% \mathrm{CO}_{2}$. At the end of the incubation period, culture media from the four wells were collected and pooled and cells removed by centrifugation. The supernatant fractions were kept at $-20{ }^{\circ} \mathrm{C}$ until assayed. Cytokine concentrations in the supernatant fractions were measured in duplicate by ELISA utilizing commercially prepared kits (Bender MedSystems, Vienna, Austria). The detection limits of the IFN- $\gamma$, IL-2, TNF- $\alpha$, IL- 6 and IL-1 $\beta$ assays were $1 \cdot 5,15 \cdot 0,5 \cdot 0,1.4$ and $1.0 \mathrm{pg} / \mathrm{ml}$ respectively.

\section{Statistics}

All statistical analyses were performed using SPSS software (version 8.0, SPSS Inc., Chicago, IL). Logarithmic transformation of the variables measuring cytokine production was calculated in order to obtain normality in the distribution of these data. Student's $t$ test with Levene's test for equality of variances was then used to compare mean cytokine production between $\mathrm{AN}$ and control groups. To assess the differences observed in the AN patients between admission and discharge, Student's $t$ test for paired data was used. Finally, Pearson's correlation coefficients were calculated to find significant relationships between immunological function and individual characteristics of the subjects. Median and interquartile ranges are presented to clarify the range of production for each cytokine.

\section{Results}

Demographic characteristics of the subjects are presented in Table 1. There was no significant difference in age between patients and control subjects. Patients were significantly shorter than control subjects, although the mean height-for-age values in both groups were on about the 50th percentile of the Spanish Growth Reference Charts (Hernandez et al. 1988). The onset of AN had occurred 1.45 (SD 0.76) years prior to entering the current study. All patients were amenorrhoeic and nine presented primary 
Table 1. Demographic characteristics of controls and anorexia nervosa (AN) patients upon hospital admission and at discharge

(Mean values and standard deviations)

\begin{tabular}{|c|c|c|c|c|c|c|}
\hline & \multicolumn{2}{|c|}{ Controls ( $n$ 35) } & \multicolumn{2}{|c|}{ ANO ( $n$ 37) } & \multicolumn{2}{|c|}{ AN1 ( $n$ 37) } \\
\hline & Mean & SD & Mean & SD & Mean & SD \\
\hline Age (years) & $15 \cdot 43$ & 1.27 & $15 \cdot 82$ & 1.46 & $15 \cdot 87$ & 1.46 \\
\hline Weight $(\mathrm{kg})$ & 54.41 & $9 \cdot 16$ & $38 \cdot 84^{\star \star *}$ & 4.65 & 42.93***†† & 3.83 \\
\hline Height (m) & 1.623 & 0.055 & $1.588^{\star \star}$ & 0.051 & $158 \cdot 8^{\star \star}$ & 0.051 \\
\hline$\%$ Ideal body weight & 96.07 & $11 \cdot 81$ & $75 \cdot 21^{\star \star \star}$ & 7.67 & $83.66^{\star \star \star}+\dagger \dagger$ & 6.31 \\
\hline BMI & $20 \cdot 58$ & $2 \cdot 70$ & $15 \cdot 38^{\star \star \star}$ & 1.44 & $17 \cdot 05^{\star \star \star}+† \dagger$ & 1.01 \\
\hline
\end{tabular}

AN0, on admission to hospital; AN1, on discharge from hospital.

Mean values were significantly different from those of the contol group (Student's $t$ test): ${ }^{\star \star} P<0.01,{ }^{\star \star \star} P<0.001$.

Mean values were significantly different from those of ANO (Student's $t$ test): $\dagger \dagger \dagger P<0.001$.

amenorrhoea. The mean duration of the in-patient period was 31 (SD 8) d. Weight, \% ideal body weight and BMI were significantly lower in anorexic individuals in ANO. One month of in-patient treatment significantly improved all these variables, although they did not reach control values. BMI increased from $-2 \cdot 2 \mathrm{SD}$ of the healthy, age-matched population (Hernandez et al. 1988) in AN0 to $-1.5 \mathrm{SD}$ in AN1. According to the classification of Llewellyn-Jones \& Abraham, (1984), BMI values increased from the emaciation threshold (BMI $<15)$ in AN0 to values that reflect an underweight status $(\mathrm{BMI}<$ 19) in AN1.

Results on the capacity of PBMC to produce cytokines after PHA stimulation are presented in Table 2. Very high inter-individual variations were observed in the production of each of the cytokines measured. The median and the interquartile range (Table 2, Fig. 1) are more useful than the mean value of the variable, as some extreme cytokine production values were obtained in AN patients. IFN- $\gamma$ and IL-2 production by cells from AN patients at admission were similar to those of the control group. At discharge, IFN- $\gamma$ production by cells from AN patients was significantly $(P<0 \cdot 047)$ lower than control values, while IL-2 production remained unmodified. Tumour necrosis factor- $\alpha$ and IL- 6 production by cells from AN patients at admission and discharge were significantly $(P<0.001)$ lower than those observed in the control group. However, IL1- $\beta$ production was significantly $(P<0.001)$ higher in AN patients at admission and discharge than in control subjects. No significant differences between cytokine production at admission and discharge were noted. Significant correlations were found between the duration of the illness previous to hospital admission and the production of IL-1 $\beta \quad(r-0.47$, $P=0.003)$ and IFN- $\gamma(r \quad 0.32, P=0.048)$ observed in AN0. No correlations were found between cytokine production and other demographic characteristics such as age or \% ideal body weight.

\section{Discussion}

The balance between immunomodulatory mediators on the one hand, and the capacity of T lymphocytes to respond to these mediators on the other hand, is an important element in the complex process of $\mathrm{T}$ cell activation and clonal expansion, and thus in the immune response. Dysregulated cytokine production, mainly through an altered proinflammatory cytokine secretory pattern, is shown in the present study.

Highly significant inter-individual variations in the production of cytokines by purified mononuclear cells from healthy subjects have been reported (Yaqoob et al. 1999). This diversity was also observed in the current study, although variability tended to be higher in the AN group than in control individuals. Some important features with a potential effect on cytokine production may differ among patients, such as drug consumption, the selfimposed weight-control mechanisms and neuroendocrine

Table 2. In vitro cytokine production by peripheral blood mononuclear cells (pg/ml) in controls and anorexia nervosa (AN) patients upon hospital admission and at discharge*

(Median values and interquartile ranges)

\begin{tabular}{|c|c|c|c|c|c|c|c|c|}
\hline & \multicolumn{2}{|c|}{ Controls (n 35) } & \multicolumn{3}{|c|}{ ANO $(n 37) \dagger$} & \multicolumn{3}{|c|}{ AN1 $(n 37) \dagger$} \\
\hline & Median & Interquartile range & Median & Interquartile range & $P$ value $\ddagger$ & Median & Interquartile range & $P$ value $\neq$ \\
\hline IFN- $\gamma$ & 322.0 & $133 \cdot 0-701 \cdot 4$ & $199 \cdot 7$ & $27 \cdot 5-977 \cdot 1$ & NS & 80.4 & $29 \cdot 1-464 \cdot 5$ & 0.047 \\
\hline IL-2 & 117.9 & $61 \cdot 1-236.5$ & 96.8 & $80 \cdot 7-167.7$ & NS & $96 \cdot 8$ & $85 \cdot 9-149 \cdot 1$ & NS \\
\hline TNF- $\alpha$ & $132 \cdot 2$ & $52 \cdot 5-246 \cdot 8$ & 18.4 & $6 \cdot 4-67 \cdot 1$ & $<0.001$ & 8.4 & $1.0-57.8$ & $<0.001$ \\
\hline IL-6 & 2383.6 & $1031 \cdot 6-4200 \cdot 0$ & $852 \cdot 2$ & $474.5-1193.2$ & $<0.001$ & $621 \cdot 8$ & $164 \cdot 6-1299 \cdot 2$ & $<0.001$ \\
\hline IL-1 $\beta$ & 30.9 & $19 \cdot 8-81.5$ & 888.3 & $378.5-1707 \cdot 1$ & $<0.001$ & 709.5 & $469 \cdot 5-1846 \cdot 8$ & $<0.001$ \\
\hline
\end{tabular}

ANO, on admission to hospital; AN1, on discharge from hospital; IFN, interferon; IL, interleukin.

${ }^{*}$ For details of subjects and methods, see. Table 1 and p. 184.

† No significant differences were observed between ANO and AN1.

$\ddagger$ Student's $t$ test of the log-transformed variable (values were compared with those of the control group). 
(a)

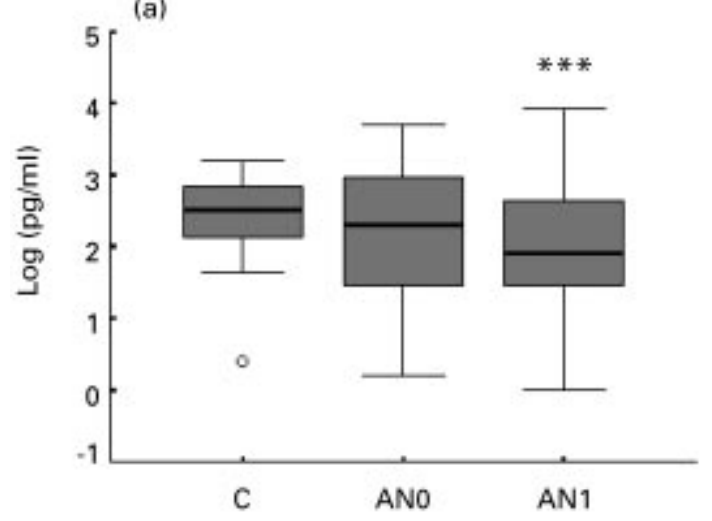

(b)

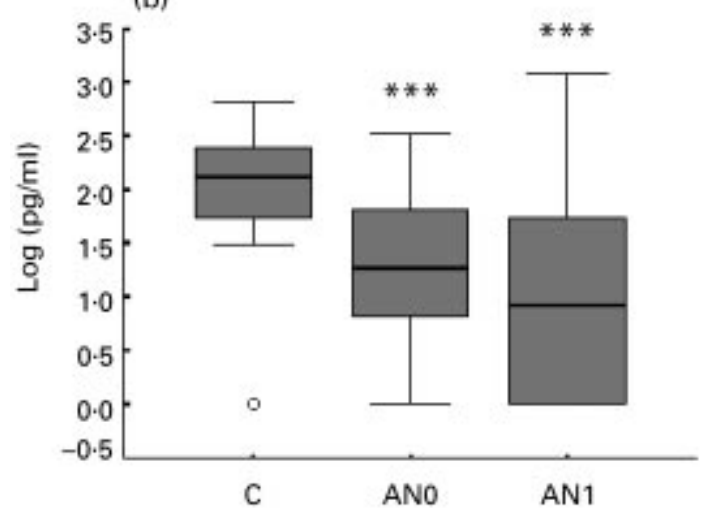

(c)

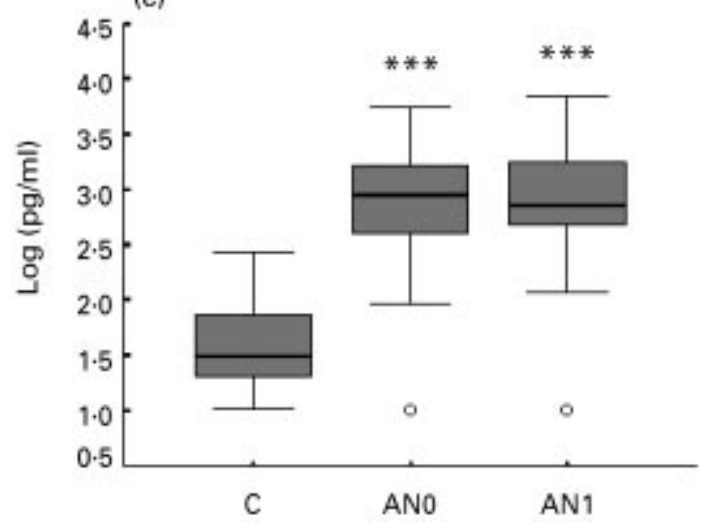

(d)

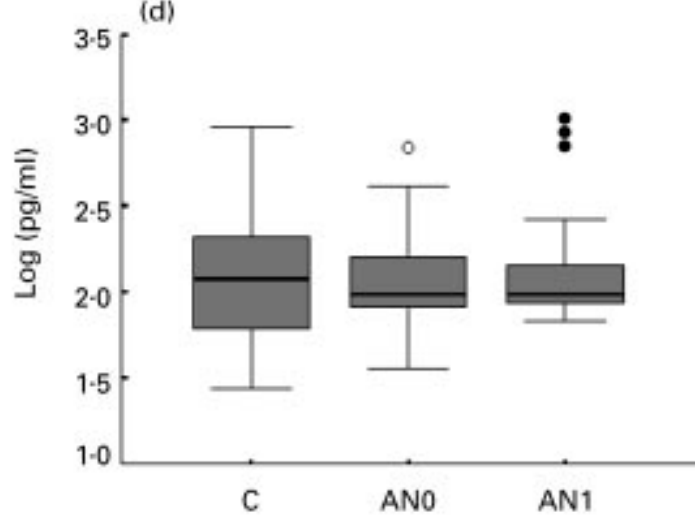

(e)

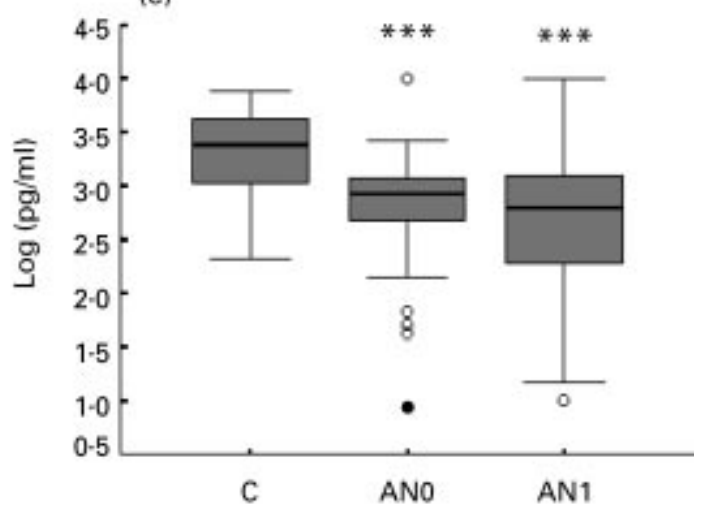

Fig. 1. Box plots showing median and interquartile ranges of cytokines production by peripheral blood mononuclear cells of control subjects (C) and anorexia nervosa (AN) patients on hospital admission (AN0) and on discharge from hospital (AN1). The log transformed values are represented. (a), interferon- $\gamma$ (C $n$ 35, AN0 $n$ 36, AN1 $n 36$ ); (b), tumour necrosis factor $\alpha$ (C $n$ 35, ANo $n 36$, AN1 $n$ 36); (c), interleukin-1 $\beta$ (C $n$ 35, ANO $n$ 35, AN1 $n$ 35); (d), interleukin 2 (C $n$ 35, ANO $n$ 35, AN1 $n$ 35); (e), interleukin 6 (C $n$ 35, AN0 $n$ 37, AN1 $n$ 37). For details of subjects and procedures, see Table 1 and p. 184. (O), Outlier (cases with values between 1.5 and 3 interquartile ranges from upper or lower edge of the box); (๑), extreme value (cases with values more than three interquartile ranges from upper or lower edge of box). Mean values were significantly different from those of the control group: ${ }^{* \star *} P<0.001$.

alterations (Marcos, 1997). Although the importance of these cannot be underestimated, we did not attempt to consider them in this study, since little is known about the relative or cumulative influence of any of them. Experiments in vivo and in vitro have shown that acute administration of fluoxetine (Pellegrino \& Bayer, 1998) or diazepam (benzodiazepine) (Pawlikowski et al. 1988) results in a decrease in mitogen-induced lymphocyte proliferation. There are few studies reporting the effect of fluoxetine on cytokine production. In rats treated daily with fluoxetine for 5 weeks, lipopolysaccharide-induced expression of TNF- $\alpha$ and IL- $1 \beta$ in the spleen was not altered (Yirmiya et al. 2001). However, benzodiazepines would be more likely to inhibit proinflammatory cytokine production (Fruscella et al. 2001; Nawrocka et al. 2001). Another factor that might influence the response observed in our patients is a variable sensitivity to the stimulus in different individuals. Since a single concentration of mitogen was used rather than a series of concentrations, the differences found between patients and controls could 
reflect an altered sensitivity to the stimulus and not necessarily an altered production capacity.

Although lymphocytes of AN patients display major functional defects (Pomeroy et al. 1994), no alteration in the IFN- $\gamma$ and IL-2 production by cells from adolescent AN patients at the moment of their admission to hospital was found in the current study. However, after 1 month of in-patient treatment study individuals (AN1) displayed lower IFN- $\gamma$ production than the control group. Previous results by Schattner et al. (1990b) and Polack et al. (1993) point out an apparent inhibition of PHA-induced IFN- $\gamma$ production by PBMC of $\mathrm{AN}$ patients in relation to that of age-matched controls. However, Schattner et al. (1990a) also report the absence of substantial differences in $2^{\prime}-5^{\prime}$ oligo-adenylate synthetase activity and, therefore, in the potential of IFN- $\gamma$ system activation in vivo, between PBMC of AN patients and those of healthy controls consuming a normal diet. Schattner et al. (1990b) reported normalization of IFN- $\gamma$ secretion after refeeding; the long period of time between the first and second evaluations (9 months) and the substantial recovery of patients in that study with regard to all diagnostic criteria could account for this outcome. Our present subjects, however, were still in a poor physical condition at discharge and the IFN- $\gamma$ values observed in the current study could be related to the short duration of the follow-up, which was limited to the in-patient treatment period.

The normal results obtained for IL-2 production observed in AN patients do not coincide with the results of Bessler et al. (1993), who found that AN patients of similar age to those in the present study displayed a lower PHA-stimulated production of IL- 2 by PBMC. However, there is one important difference between the patients in these studies. While in Bessler's study subjects had been drug-free for at least 2 months, most of our patients were undergoing pharmacological therapy and psychotherapy. Bessler et al. (1993) suggest that stress and depression in their patients, as well as nutritional deficiencies, could be responsible for impaired cytokine synthesis.

With regard to the other cytokines measured, there have been few studies carried out utilizing PHA as a stimulus. No differences in TNF- $\alpha$ values were found between AN patients and control individuals in one study under these conditions (Schattner et al. 1990a). However, in the present study TNF- $\alpha$ production was lower in AN patients than in control individuals. Other studies using lipopolysaccharide as a stimulus have also reported lower TNF- $\alpha$ production by PBMC from AN patients (Schattner et al. 1990a; Nagata et al. 1999), while spontaneous production, without a stimulus, has been shown to be higher in AN subjects than in controls (Schattner et al. 1990b; Allende et al. 1998). In this sense, it has been suggested that cells which have already been activated to produce a cytokine may respond poorly to further stimulation (Preble et al. 1983; Bowen et al. 1985; Magilavy \& Rothstein, 1988). Although we do not have results on spontaneous production or serum cytokine levels in our patients, this mechanism could explain why cells from our patients displayed lower TNF- $\alpha$ production after PHA stimulation than those from control individuals.

In the same way, and coinciding with our present results, mitogen-stimulated PBMC cells of AN patients were previously found to produce lower amounts of IL-6 than those from healthy subjects (Nagata et al. 1999). In addition, underweight AN patients exhibit higher serum IL-6 values than control individuals (Pomeroy et al. 1994). These observations point out again that a normal in vitro response might be lacking when cells have been pre-stimulated in vivo. A possible role for medication on IL-6 and TNF- $\alpha$ production should also be considered. For example, benzodiazepine tricyclic derivatives inhibit in vivo IL-6 production in mice (Fruscella et al. 2001). Inhibitory properties have also been found in vitro with regard to lipopolysaccharide-induced TNF- $\alpha$ production (Nawrocka et al. 2001).

Some of the endocrine changes reported in AN patients, such as reduced sex hormone levels and increased cortisol and $\beta$-endorphin secretion (Walsh et al. 1987; Tepper et al. 1992), have been shown to affect the cellular immune function (Blalock et al. 1985). However, the relationship between the neuroendocrine and the immune systems is bi-directional. Thus, TNF- $\alpha$ and IL- 1 are proinflammatory cytokines that are capable of activating the hypothalamicpituitary-adrenal axis and have a stimulatory effect on adrenal corticosterone secretion in vitro (Vaisman \& Hahn, 1991; Day \& Akil, 1996). In turn, IL-1 expression may be suppressed by glucocorticoids and their receptors in monocytes, which are capable of physiologically controlling the IL-1-IL-1 receptor antagonist system during inflammatory or immune processes. Thus, IL-1 $\beta$ production in vitro could be very different from production in vivo, where a broad array of mediators could be affecting the system and there is a lack of stimulation of IL-1 $\beta$ production in the body. In addition, the negative correlation found between IL-1 $\beta$ production and the duration of the illness indicates that patients with a longer illness period tend to show lower production of the cytokine in vitro. This means that their values are closer to control values, since the mean production is increased in AN patients compared with controls. This could reflect a long-term adaptation process such that, in longer illnesses, different compensatory mechanisms are taking place in comparison with the mechanisms triggered in the short term.

In conclusion, the results suggest that the changes in the secretion pattern of proinflammatory cytokines could be relevant in explaining some immune function findings in underweight AN patients. These mechanisms involving changes in cytokine production might play a role in the adaptive process to this atypical situation of malnutrition. Findings which increase our knowledge about the bi-directional communication pathways between the neuroendocrine and immune systems will enable further studies trying to define the role of cytokines in the pathogenesis of this syndrome.

\section{Acknowledgements}

The authors of this work wish to acknowledge the financial support of the Clinical Enteral Nutrition Division of NUTRICIA, Madrid. 


\section{References}

Allende LM, Corell A, Manzanares J, Madruga D, Marcos A, Madrono A, Lopez-Goyanes A, Garcia-Perez MA, Moreno JM, Rodrigo M, Sanz F \& Arnaiz-Villena A (1998) Immunodeficiency associated with anorexia nervosa is secondary and improves after refeeding. Immunology 94, 543-551.

American Psychiatric Association (1994) Diagnostic and Statistical Manual of Mental Disorders, 4th ed., Washington, DC: APA.

Armstrong-Esther CA, Lacey JH, Crisp AH \& Bryant TN (1978) An investigation of the immune response of patients suffering from anorexia nervosa. Postgraduate Medical Journal 54, 395-399.

Bessler H, Karp L, Notti I, Apter A, Tyano S, Djaldetti M \& Weizman R (1993) Cytokine production in anorexia nervosa. Clinical Neuropharmacology 16, 237-243.

Blalock JE, Harbour-McMenamin D \& Smith EM (1985) Peptide hormones shared by the neuroendocrine and immunologic systems. Journal of Immunology 135, 858s-861s.

Bowen DL, Lane HC \& Fauci AS (1985) Immunopathogenesis of the acquired immunodeficiency syndrome. Annals of Internal Medicine 103, 704-709.

Cason J, Ainley CC, Volstencroft RA, Norton KRW \& Thompson RPH (1986) Cell mediated immunity in anorexia nervosa. Clinical and Experimental Immunology 64, 370-375.

Chandra RK \& Kumari S (1994) Effects of nutrition on the immune system. Nutrition 10, 207-210.

Day HE \& Akil H (1996) Differential pattern of c-fos mRNA in rat brain following central and systemic administration of interleukin-1 beta: implications for mechanism of action. Neuroendocrinology 63, 207-218.

Fruscella P, Sottocorno M, Di Braccio M, Diomede L, Piccardi N, Cagnotto A, Grossi G, Romano M, Mennini T \& Roma G (2001) 1,5-Benzodiazepine tricyclic derivatives exerting antiinflammatory effects in mice by inhibiting interleukin-6 and prostaglandin E(2)production. Pharmacology Research 43, $445-452$

Golla AG, Larson LA, Anderson CF, Lucas AR, Wilson WR \& Tomasi TB (1981) Immunological assessment of patients with anorexia nervosa. American Journal of Clinical Nutrition 34, 2756-2762.

Hernandez M, Castellet J, Narvaíza JL, Rincón JM, Ruiz E, Sánchez E, Sobradillo B \& Zuzimendi A (1988) Curvas y Tablas de Crecimiento (Growth Charts and Tables) [Faustino Orbegozo Foundation, editors]. Madrid: Carsi.

Holden RJ \& Pakula IS (1996) The role of tumour necrosis factor$\alpha$ in the pathogenesis of anorexia and bulimia nervosa, cancer cachexia and obesity. Medical Hypothesis 47, 423-438.

Llewellyn-Jones D \& Abraham SF (1984) Quetelet index in the diagnosis of anorexia nervosa. British Medical Journal 288 , 1800 .

Magilavy DB \& Rothstein JL (1988) Spontaneous production of tumor necrosis factor $\alpha$ by Kupffer cells of MRL/lpr mice. Journal of Experimental Medicine 168, 789-794.

Marcos A (1997) The immune system in eating disorders: An overview. Nutrition 13, 853-862.

Marcos A, Varela P, Santacruz I, Muñoz-Velez A \& Morande G (1993) Nutritional status and immunocompetence in eating disorders. A comparative study. European Journal of Clinical Nutrition 47, 787-793.

Nagata T, Tobitani W, Kiriike N, Iketani $\mathrm{T}$ \& Yamagami S (1999) Capacity to produce cytokines during weight restoration in patients with anorexia nervosa. Psychosomatic Medicine 61, $371-377$.

Nawrocka W, Sztuba B \& Zimecki M (2001) Synthesis and immunotropic properties of 5-substituted 1,5-benzodiazepin-2ones derivatives in cultures of human peripheral blood cells, Part III. Archiv der Pharmazie 334, 11-16.

Pawlikowski M, Lyson K, Kunert-Radek J \& Stepien H (1988) Effect of benzodiazepines on the proliferation of mouse spleen lymphocytes in vitro. Journal of Neural Transmission 73, 161-166.

Pellegrino TC \& Bayer BM (1998) Modulation of immune cell function following fluoxetine administration in rats. Pharmacology, Biochemistry and Behaviour 59, 151-157.

Polack E, Nahmod VE, Emeric-Sauval E, Bello M, Costas M, Finkielman S \& Arzt E (1993) Low lymphocyte interferongamma production and variable proliferative response in anorexia nervosa patients. Journal of Clinical Immunology 13, 445-451.

Pomeroy C, Eckert E, Hu S, Eiken B, Mentink M, Crosby RD \& Chao CC (1994) Role of interleukin-6 and transforming growth factor- $\beta$ in anorexia nervosa. Biological Psychiatry 36, $836-839$.

Preble OT, Rothko K, Klippel JH, Friedman RM \& Johnston MI (1983) Interferon-induced $2^{\prime}-5^{\prime}$ adenylate synthetase in vivo and interferon production in vitro by lymphocytes from systemic lupus erythematosus patients with and without circulating interferon. Journal of Experimental Medicine 157, 2140-2146.

Schattner A, Steinbock M, Tepper R, Schonfeld A, Vaisman N \& Hahn T (1990a) Tumour necrosis factor production and cellmediated immunity in anorexia nervosa. Clinical and Experimental Immunology 79, 62-66.

Schattner A, Tepper R, Steinbock M, Vaisman N, Hahn T \& Schonfeld A (1990b) TNF, interferon- $\gamma$, and cell-mediated cytotoxicity in anorexia nervosa; effect of refeeding. Journal of Clinical and Laboratory Immunology 32, 183-184.

Sullivan PF (1995) Mortality in anorexia nervosa. American Journal of Psychiatry 152, 1073-1074.

Tepper R, Weizman A, Apter A, Tyano S \& Beyth Y (1992) Elevated plasma immunoreactive beta-endorphin in anorexia nervosa. Clinical Neuropharmacology 15, 387-391.

Vaisman N \& Hahn T (1991) Tumor necrosis factor- $\alpha$ and anorexia - cause or effect? Metabolism 40, 720-723.

Vaisman N, Barak Y, Hahn T, Karov Y, Malach L \& Barak V (1996) Defective in vitro granulopoiesis in patients with anorexia nervosa. Pediatric Research 40, 108-111.

Varela P, Marcos A, Muñoz-Velez A, Santacruz I \& Requejo A (1988) Delayed hypersensitivity skin test in anorexia nervosa and bulimia. Medical Science Research 16, 1135.

Wade S, Bleiberg F, Mossé A, Lubetzki J, Flavigny H, Chapuis P, Roceh D, Lemonnier D \& Dardenne M (1985) Thymulin (ZnFacteur Thymique Serique) activity in anorexia nervosa patients. American Journal of Clinical Nutrition 42, 275-280.

Walsh BT, Roose SP, Katz JL, Dyrenfurth I, Wright L, Vande Wiele R \& Glassman AH (1987) Hypothalamic-pituitaryadrenal-cortical activity in anorexia nervosa and bulimia. Psychoneuroendocrinology 12, 131-140.

Yaqoob P, Newsholme EA \& Calder PC (1999) Comparation of cytokine production in cultures of whole human blood and purified mononuclear cells. Cytokine 11, 600-605.

Yirmiya R, Pollak Y, Barak O, Avitsur R, Ovadia H, Bette M, Weihe E \& Weinfeld J (2001) Effects of antidepressant drugs on the behavioral and physiological responses to lipopolysaccharide (LPS) in rodents. Neuropsychopharmacology 24, $531-544$ 